\title{
Evidence of Exchange-Bias-Like Phenomenon in GdFe/TbFe/GdFe Domain Wall Junctions
}

\author{
S. Mangin, ${ }^{1}$ G. Marchal, ${ }^{1}$ and B. Barbara ${ }^{2}$ \\ ${ }^{1}$ Laboratoire de Physique des Matériaux, Université H. Poincaré, BP 239, 54506 Vandoeuvre les Nancy Cedex, France \\ ${ }^{2}$ Laboratoire de Magnétisme Louis Néel, CNRS, BP 166, 38042 Grenoble Cedex 9, France
}

(Received 12 June 1998; revised manuscript received 31 December 1998)

\begin{abstract}
An exchange-bias-like phenomenon has been observed in amorphous $\mathrm{GdFe} / \mathrm{TbFe} / \mathrm{GdFe}$ trilayers called domain wall junctions. They show classical ferromagnetic/antiferromagnetic behavior, and particularly, a characteristic hysteresis loop shift. The bias-like field is attributed to the occurrence of domain walls (DWs) at the interface which can be compressed or decompressed by the external field. We show convincing evidence of these DWs from ac-susceptibility measurements. An evaluation of the bias field is proposed. [S0031-9007(99)09165-6]

PACS numbers: 75.60.Ch, 75.70.-i
\end{abstract}

The phenomenon of exchange bias usually arises in samples composed of ferromagnetic $(F)$ and antiferromagnetic (AF) materials $[1,2]$ and is typically observed in $\mathrm{F} /$ AF bilayers. Its defining characteristic is, after cooling of the sample in a high positive field, a shift of the hysteresis loop from $H=0$ to a bias field $H_{E}$. This bias is usually negative, but can also be positive [3]. The fundamental origin of this field is not perfectly clear, and it is still the object of discussions [1,4-6]. $H_{E}$ is generally considered to be due to a magnetic interface coupling between the AF and $\mathrm{F}$ layers and is given by

$$
H_{E}=\frac{\Delta \sigma}{2 M_{\mathrm{F}} t_{\mathrm{F}}},
$$

where $M_{\mathrm{F}}$ and $t_{\mathrm{F}}$ are the saturation magnetization and the thickness of the $\mathrm{F}$ layer, respectively, and $\Delta \sigma$ is the interface energy arising when the $\mathrm{F}$ moments reverse. Equation (1) expresses the balance between the interface and the Zeeman energies. A domain wall (DW) extends the influence of the $\mathrm{F}$ magnetization reversal into the AF layer, lowering the total interface energy between the two magnetic systems [5].

At present the bias field effect is of great interest because of the related theoretical problems of interface coupling and because of the recent technological progresses in magnetoelectronics [7]. In fact, the exchange field does not necessarily have to be due to the presence of an antiferromagnetic layer. The only requirement is that a DW develops at the interface between $F$ and a second magnetic material, which is insensitive to the external field, either because its net magnetization is zero as in AF, or because its anisotropy is so strong that its magnetic state is insensitive to a weak field.

The sperimagnetic rare earth transition-metal amorphous alloys $(s p$-RE-TM) can replace the AF layer. Indeed, in these materials, the anisotropy (due to RE) is very strong, and the field cooling induces a remanent magnetization [8]. The reversal of the magnetization of the alloy requires a strong magnetic field $(1 \mathrm{~T})$, and the hysteresis loops are almost square, meaning that the magnetization is on average strongly constrained along the cooling field direction. A difference between the F/AF and F/sp-RETM systems, where $F$ is a soft ferromagnetic layer, is that in F/AF, the interface DW develops rather inside the AF layer, whereas in F/sp-RE-TM, because of the strong anisotropy of the RE, the DW rotation is largely contained within the F layer. Another difference is that the sign of exchange interactions at the interface between the two layers depends on the interface roughness in the F/AF systems [5] and may induce magnetic frustrated states at the interface, whereas in F/sp-RE-TM it is not the case.

In this Letter, we present the exchange-bias-like behavior of the bilayer system $\mathrm{GdFe} / \mathrm{TbFe}$ and we apply it to $\operatorname{GdFe}\left(e_{1}\right) / \operatorname{TbFe}(e) / \operatorname{GdFe}\left(e_{2}\right)$ which we call a domain wall junction (DWJ) [9]. TbFe is the $s p$-RE-TM, and GdFe is the soft magnetic material. The compositions of the alloys are $\mathrm{Gd}_{62} \mathrm{Fe}_{38}$ and $\mathrm{Tb}_{55} \mathrm{Fe}_{45}$. The materials are sperimagnetic, but, because of the high magnetic moment of the REs, the net magnetizations are along those of the RE sublattices. The iron magnetic moments in both layers are antiparallel to the net magnetization, and they control the ferromagnetic interface coupling between the GdFe and $\mathrm{TbFe}$ layers. The magnetic softness of GdFe is due to the specific electronic configuration of gadolinium, which presents a half filled $4 f$ shell and thus an isotropic $S$ state character.

This $\operatorname{GdFe}\left(e_{1}\right) / \operatorname{TbFe}(e) / \operatorname{GdFe}\left(e_{2}\right)$ system was originally prepared to study the nucleation of a DW in one of the GdFe layers and its propagation through the $\mathrm{TbFe}$ layer which constitutes a planar potential energy barrier. An in-plane uniaxial anisotropy occurs spontaneously in the GdFe layer [10], and, consequently, the DWs are very well defined Bloch walls. Actually, the device works in unsymmetrical samples where $e_{1}>e_{2}$ (typically $e_{1}=1000 \AA$ and $e_{2}=500 \AA$ ). In such samples previously saturated along the $+z$ direction, the nucleation of reversed magnetization starts from the outer part of the GdFe(1000 $\mathrm{A})$ layer [Fig. 1(a)] at a nucleation field $H_{n 1}$, with the formation of a DW. The DW is at first blocked by the TbFe layer, compresses against this layer, and then propagates 
a)

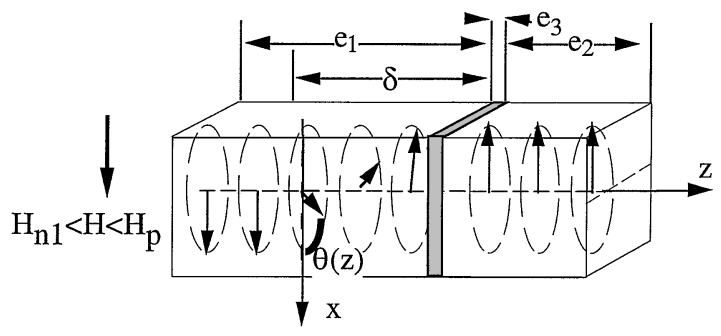

b)

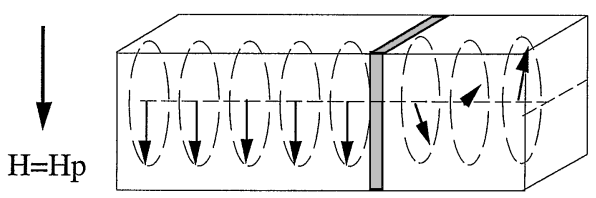

c)

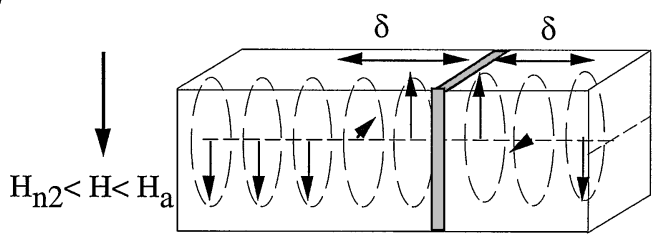

FIG. 1. Magnetic configurations in the trilayer: (a) For $H_{n 1}<H<H_{p}$ the DW is compressed against the TbFe layer. (b) At $H=H_{p}$ the outer part of the TbFe layer magnetization is reversed and the DW propagates into the thinner GdFe layer. (c) At $H=H_{n 2}$ a second DW is nucleated from the outer part of the thinner GdFe layer.

from the $\operatorname{GdFe}(1000 \AA)$ layer to the $\operatorname{GdFe}(500 \AA)$ layer because of the TbFe's magnetization reversal at the propagation field $H_{p}(T)$ [Fig. 1(b)]. The propagation of the first DW through the TbFe barrier can be bypassed if a second DW forms at the outer surface of the $\operatorname{GdFe}(500 \AA)$ layer. This occurs at $H_{n 2}$ [Fig. 1(c)]. In that case, the two DWs are blocked and compressed against the TbFe layer before simultaneous annihilation at the annihilation field $H_{a}$. In a previous paper [11] it was shown that $H_{p}(T)$ increases with decreasing temperature indicating that the phenomenon was thermally activated. Of course, the range of $H_{p}(T)$ is limited by $H_{n 1}$ and $H_{n 2}$ and increases with the thickness of the $\mathrm{TbFe}$ layer.

In this Letter, we focus on the compression and decompression of the DW against the TbFe layer which is an exchange-bias-like phenomenon. The data are from a $\operatorname{GdFe}(1000 \AA) / \operatorname{TbFe}(9 \AA) / \operatorname{GdFe}(500 \AA)$ sample. The Curie temperature of the system is $T_{c}=325 \mathrm{~K}$. At $27 \mathrm{~K}$ the nucleation occurs in the $\operatorname{GdFe}(1000 \AA)$ layer at $H_{n 1} \approx$ $18 \mathrm{Oe}$, and the propagation of the DW through the TbFe layer occurs around $H_{p}=50 \mathrm{Oe}$. At $10 \mathrm{~K}$, the propagation field is too high, and the first nucleation at $H_{n 1} \approx$ 18 Oe in the $\operatorname{GdFe}(1000 \AA)$ layer is followed by nucleation in the $\operatorname{GdFe}(500 \AA)$ layer at $H_{n 2} \approx 60$ Oe. After these two nucleations, the TbFe layer is squeezed between two $\pi$-DWs. These features are shown in Figs. 2 and 3: from the net magnetization $M$ in 2(a) and 3(a), and from the ac susceptibility $\chi_{\text {ac }}$ in 2(b) and 3(b), measured after cooling of the sample from $100 \mathrm{~K}$ in a +1000 Oe field. The ac susceptibility $\left(\chi_{\mathrm{ac}}\right)$ was measured with a $10 \mathrm{kHz}$, 0.25 Oe field parallel to the static field. We observed that $\chi_{\text {ac }}$ was almost independent of the frequency probably because mean DW oscillation modes lie well above these frequencies.

In Fig. $2(T=27 \mathrm{~K})$, the remanent magnetization is equal to the saturation magnetization. The first step (at $\left.H_{n 1}\right)$ is due to the nucleation of reversed magnetization in the $\operatorname{GdFe}(1000 \AA)$ layer as schematized in Fig. 1(a). The second one (at $H_{p}$ ) results from the propagation of the DW through the TbFe layer [Fig. 1(b)]. At $H>H_{p}, M$ drops

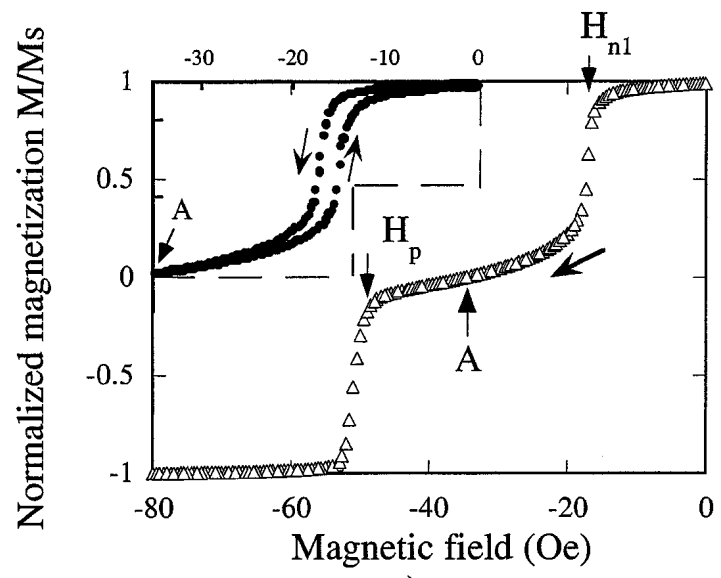

a)

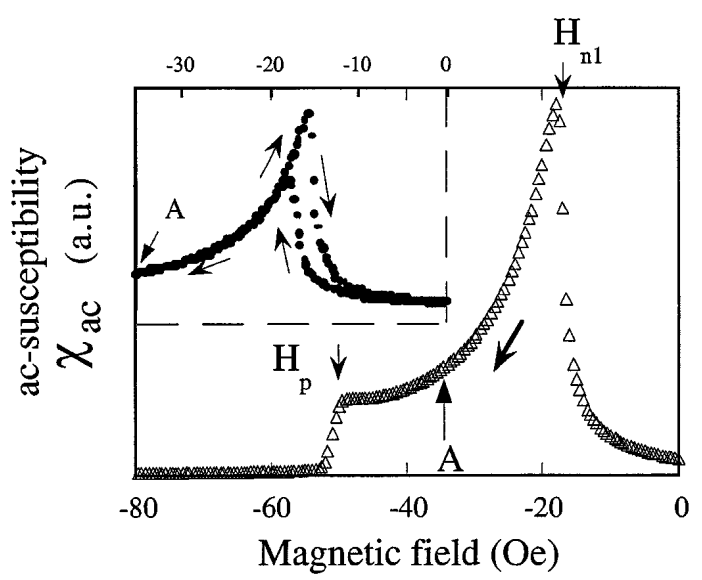

b)

FIG. 2. (a) Normalized magnetization for the $\operatorname{GdFe}(1000 \AA) /$ $\operatorname{TbFe}(9 \AA) / \operatorname{GdFe}(500 \AA)$ sample, at $27 \mathrm{~K}$. The sample has been first saturated at $100 \mathrm{~K}$ with a 1000 Oe field and the magnetization measured from +1000 Oe to -1000 Oe (main figure), then from +1000 Oe to $H=-35$ Oe (point $A$ ) and finally from $H=-35$ Oe to $H=+100 \mathrm{Oe}$ (inset). The saturated magnetization $M_{s}$ is the magnetization at $27 \mathrm{~K}$ and +1000 Oe. (b) ac susceptibility under the same conditions with a $0.25 \mathrm{Oe}, 10^{4} \mathrm{~Hz}$ field parallel to the static field. 


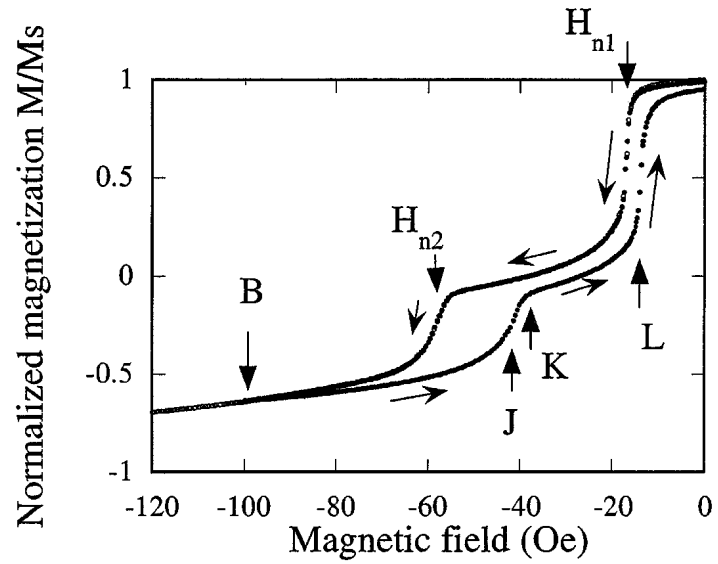

a)

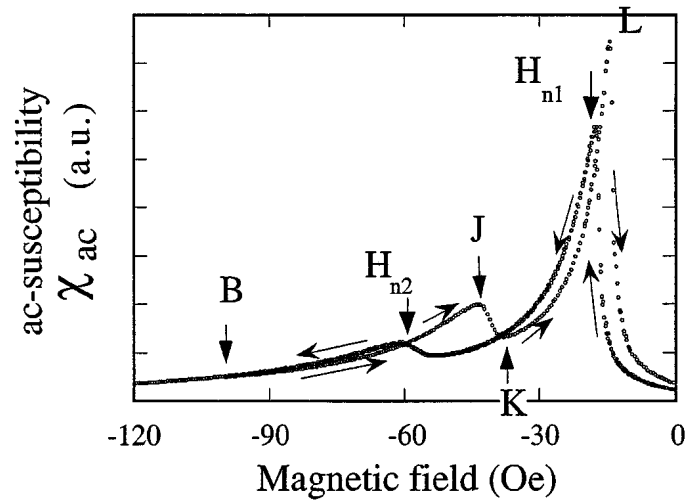

b)

FIG. 3. (a) Normalized magnetization for the $\operatorname{GdFe}(1000 \AA) /$ $\operatorname{TbFe}(9 \AA) / \mathrm{GdFe}(500 \AA)$ sample, at $10 \mathrm{~K}$. The sample has been first saturated at $100 \mathrm{~K}$ with a $1000 \mathrm{Oe}$ field and the magnetization measured from $H=+1000$ Oe to $H=$ -1000 Oe. A minor loop has been collected from $H=$ -100 Oe. (b) ac susceptibility under the same conditions with a $0.25 \mathrm{Oe}, 10^{4} \mathrm{~Hz}$ field parallel to the static field.

to $-M_{s}$, because the DW disappears and the DWJ is fully magnetized. Between $H_{n 1}$ and $H_{p}$ there is a DW at the $\mathrm{GdFe} / \mathrm{TbFe}$ interface located on the GdFe side. This DW is compressed by the pressure of the applied field against the TbFe barrier layer whose magnetization is constrained by the anisotropy. The DW is very well seen from $\chi_{\mathrm{ac}}$. Indeed, at low temperature (well below $T_{c}$ ), the transverse ac susceptibility must be significantly larger than the longitudinal one. The $\chi_{\text {ac }}$ signal essentially comes from the magnetic moment components perpendicular to the oscillating field. As seen in Fig. 1, such components are present only in the DW. The nucleation of the DW gives rise at $H_{n 1}$ to a sharp increase of $\chi_{\text {ac }}$, and its disappearance at $H_{p}$ leads to a drop of $\chi_{\text {ac }}$. Between $H_{n 1}$ and $H_{p}$ the susceptibility decreases because of the compression of the DW, which leaves fewer and fewer spins with a transverse component. At $10 \mathrm{~K}$ (Fig. 3), the first nucleation at $H_{n 1}$ in the $\operatorname{GdFe}(1000 \AA)$ layer is followed by a second nucleation at $H_{n 2}$ in the $\operatorname{GdFe}(500 \AA)$ layer, which gives rise to two successive peaks of the susceptibility. Between $H_{n 1}$ and $H_{n 2}$, there is compression of the first DW. From variation of the magnetization amplitude we conclude that the DW width varies from 600 to $400 \AA$. Beyond $H_{n 2}$, there is a simultaneous compression of the two DWs. $\chi_{\text {ac }}$ returns to 0 at $H_{a}=180 \mathrm{Oe}$ when the magnetization of the $\mathrm{TbFe}$ layer finally reverses.

In order to observe the decompression of the DWs, the static magnetic field has been decreased in magnitude from two regions: that in which there is only one DW (point $A$ in Fig. 2) and that in which there are two DWs (point $B$ in Fig. 3). The decompression of the DW from point $A$ gives a shifted minor cycle quite similar to those observed in $\mathrm{F} /$ AF layers. The width of the loop $\Delta H_{n 1}$ is only a few Oe, comparable to the coercitive field of a single $1000 \AA$ thick GdFe layer [11]. The variation of $\chi_{\text {ac }}$ is shown in the inset of Fig. 2(b). We observe that, after a quite reversible part, $\chi_{\text {ac }}$ continues to increase up to the drop due to the "denucleation" of the DW. This irreversibility due to the nucleation/denucleation energy barrier is certainly also associated with the differences in initial conditions: The nucleation process involves precursor magnetization reversal effects, whereas the denucleation one starts from welldefined DW.

In Fig. 3(a) we observe the decompression of the two DWs between $B$ and $J$, the disappearance of the second DW between $J$ and $K$, the decompression of the first DW between $K$ and $L$, and finally its disappearance at $L$. We essentially retrieve the saturation magnetization at $H=0$. It is clear that the shifted hysteresis loop is broader for the denucleation of the second DW, due to the interaction between the two $\pi$-DWs located on each side of the TbFe layer. The decompression and denucleation processes are confirmed by the evolution of $\chi_{\mathrm{ac}}$ [Fig. 3(b)].

Finally we can evaluate the exchange-bias-like fields $\left(H_{n 1}\right.$ as well as $\left.H_{n 2}\right)$ which in our case identify the nucleation fields. In the case of the F/sp-RE-TM system, expression (1) can certainly be used. As the DW is essentially located in the GdFe layer, $\Delta \sigma$ is then the DW energy in GdFe and $t_{\mathrm{F}}$ in (1) has to be replaced by $\left(t_{\mathrm{F}}-\delta / 2\right)$, where $\delta$ is the width of the DW. $\Delta \sigma(H)$ and $\delta(H)$ depend on the applied field $H$ which turns out to be $H_{n}$ at the nucleation. The expression of the energy of a Bloch wall compressed by an applied magnetic field can be written

$$
\begin{gathered}
E=\int_{-\infty}^{+\infty}\left[\frac{1}{2} J a^{2}\left(\frac{d \theta(z)}{d z}\right)^{2}+K \sin ^{2} \theta(z)\right. \\
\left.+M_{s} H[1-\cos \theta(z)]\right] d z
\end{gathered}
$$

where $J$ is the exchange energy, $K$ is the anisotropy constant of the material, $a$ is the mean interatomic distance, and $\theta(z)$ is the angle between the easy axis $O x$ and the spin at position $z$ [Fig. 1(a)]. In our case $M_{s}$ and $M_{\mathrm{F}}$ are equivalent. $\delta$ can be defined by the angular discontinuity 


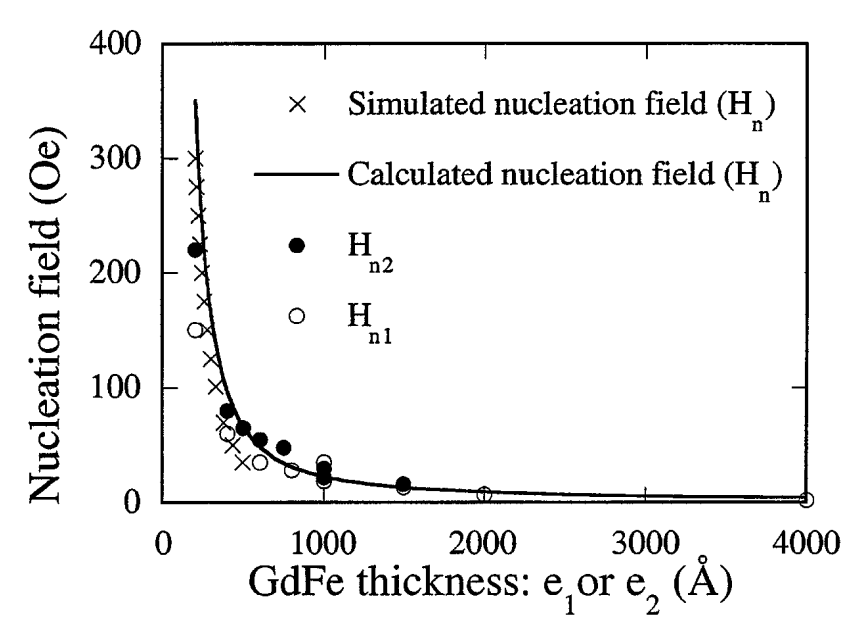

FIG. 4. Measured $\left[H_{n 1}\left(e_{1}\right)\right.$ and $\left.H_{n 2}\left(e_{2}\right)\right]$, calculated and simulated nucleation fields plotted as a function of the $\mathrm{GdFe}$ thicknesses $e_{1}$ or $e_{2}$. The nucleation fields were calculated from relation (4).

$\Delta \theta$ where $d \theta / d x$ is maximum, i.e., for $\theta=\pi / 2$, then $\delta / a=\pi / \Delta \theta$. This expression gives $\delta=\pi a \sqrt{J / K}$ and $\Delta \sigma=4 a \sqrt{J K}$ in zero field. Using the same definition under an applied field $\Delta \sigma(H)$ and $\delta(H)$ are given by

$$
\begin{aligned}
\Delta \sigma(H) & =4 a \sqrt{J\left(K+2 M_{s} H\right)} \\
\text { and } \quad \delta(H) & =\pi a \sqrt{J /\left(K+2 M_{s} H\right)} .
\end{aligned}
$$

Combining (1) and (3) leads to the critical thickness $e_{c}$ of the GdFe layer for which DW nucleation is possible under a field $H_{n}$. The expression of $e_{c}$ as a function of the nucleation field is given by

$$
\begin{aligned}
e_{c}=\frac{1}{2}[ & \frac{4 a \sqrt{J\left(K+2 M_{s} H\right)}}{H M_{s}} \\
& \left.+\pi a \sqrt{(J) /\left(K+2 M_{s} H\right)}\right] .
\end{aligned}
$$

We also performed a simulation for a linear chain of spins. We considered first neighbors exchange coupling, uniaxial anisotropy, and Zeeman energy terms. The measured nucleation fields $H_{n 1}\left(e_{1}\right)$ and $H_{n 2}\left(e_{2}\right)$ are compared to the nucleation fields obtained from the relation (4) and from the simulation in Fig. 4. A very good agree- ment is obtained with no adjustable parameters. $M_{s}=$ $1400 \mathrm{emu} / \mathrm{cm}^{3}, a=3.2 \AA$, and $K=4.2 \times 10^{4} \mathrm{erg} / \mathrm{cm}^{3}$ were determined experimentally, and the exchange constant $\left(\mathrm{Ja}^{2}\right)=15 \times 10^{-8} \mathrm{erg} / \mathrm{cm}$ has been deduced from the Hasegawa expression [12].

The simulation could be improved using a more sophisticated calculation as presented by Fang et al. [13]. It would give more information on the shape of the DWs and exhibit the very probable Bloch lines involved in the process.

In $\mathrm{GdFe} / \mathrm{TbFe}$ layers, we observed a negative exchangebias-field-like. A positive exchange-bias-like phenomena should be obtained by replacing terbium by a light RE such as $\mathrm{Nd}$, which would lead to a net antiferromagnetic coupling at the interface between the layers. A new class of bias field systems is probably emerging. Exchange spring superlattices $[14,15]$ are among these systems.

[1] W.H. Meiklejohn and C.P. Bean, Phys. Rev. 102, 1413 (1956); 105, 904 (1957).

[2] C. Tsang, N. Heiman, and Kenneth Lee, J. Appl. Phys. 52, 2471 (1981).

[3] J. Nogues, D. Lederman, T. J. Moran, and Ivan K. Schuller, Phys. Rev. Lett. 76, 4624 (1996).

[4] D. Mauri, H.C. Siegmann, P.S. Bagus, and E. Kay, J. Appl. Phys. 62, 3047 (1987).

[5] A. P. Malozemoff, Phys. Rev. B 35, 3679 (1987); J. Appl. Phys. 63, 3874 (1988); Phys. Rev. B 37, 7673 (1988).

[6] N. C. Koon, Phys. Rev. Lett. 78, 4865 (1997).

[7] For a review, see R. Coehoorn, in Magnetic Thin Films and Multilayer Systems: Physics, Analysis and Industrial Applications, edited by U. Hartmann, Springer Series in Materials Science (Springer, Heidelberg, 1996), Chap. 3.

[8] J. M. D. Coey, J. Appl. Phys. 49, 1646 (1978).

[9] L. Gunther and B. Barbara, Phys. Rev. B 49, 3926 (1994).

[10] S. Mangin et al., J. Magn. Magn. Mater. 165, 161 (1997).

[11] S. Mangin et al., Europhys. Lett. 39, 675 (1997).

[12] R. Hasegawa, J. Appl. Phys. 45, 3109 (1974).

[13] T. T. Fang and A.A. Thiele, J. Appl. Phys. 69, 4593 (1991).

[14] E. Fullerton et al., Appl. Phys. Lett. 72, 380 (1998).

[15] K. Mibu, T. Nagahama, T. Shinjo, and T. Ono, Phys. Rev. B 58, 6442 (1998). 